\title{
Correction: Respiratory management during therapeutic hypothermia for hypoxic-ischemic encephalopathy
}

\author{
Eniko Szakmar ${ }^{1,2} \cdot$ Agnes Jermendy $^{1} \cdot$ Mohamed El-Dib $^{2}$
}

Published online: 8 April 2019

(c) Springer Nature America, Inc. 2019

\section{Correction to: Journal of Perinatology \\ https://doi.org/10.1038/s41372-019-0349-2 \\ published online 11 March 2019}

The authors would like to make two corrections on the article 'Respiratory management during therapeutic hypothermia for hypoxic-ischemic encephalopathy'.

Under the 'Blood gas management during TH' paragraph there was an error in the word used to describe the reduction in $\mathrm{PCO} 2$; the sentence should read "The reduction in $\mathrm{PCO}_{2}$ is associated with a 0.012 units increase in $\mathrm{pH}$ for each $1{ }^{\circ} \mathrm{C}$ under $37^{\circ} \mathrm{C}[40,41] . "$

Under the 'Conclusions and future directions' paragraph there was an error in that a superscript character of 9 was added to the $\mathrm{PCO}_{2}$ levels; the sentence should read "To obtain optimal $\mathrm{PCO}_{2}$ levels of $40-50 \mathrm{mmHg}$, frequency and tidal volume should be kept at a minimum during mechanical ventilation [8]."

The authors apologise for the errors.

Mohamed El-Dib

mel-dib@bwh.harvard.edu

1 1st Department of Pediatrics, Semmelweis University, Budapest, Hungary

2 Department of Pediatric Newborn Medicine, Brigham and Women's Hospital, Boston, MA, USA 